\title{
Verhaltenstherapie
}

\section{Band 18, Heft 2, Juni 2008}

Nachruf

72 Steven Karger (1959-2008)

\section{Editorial}

73 Wie relevant sind alternative Behandlungsstrategien neben der Kognitiven Verhaltenstherapie?

Ehlert, U. (Zürich)

\section{Originalarbeiten}

75 Kann eine störungsorientierte Psychotherapie komorbide Symptome der Bulimia Nervosa verändern?

Tuschen-Caffier, B. (Freiburg i.Br.); Pook, M. (München); Frank, M. (Marburg)

83 Adjuvante Therapie von arterieller Hypertonie durch hochfrequentes Blutdruckbiofeedback Piesbergen, C. (München); Kircher, K. (Mühldorf/Inn); Weber, R. (Bernried)

91 Wie wirkt Expressives Schreiben? Differenzielle Wirkfaktoren des Schreibens über belastende Lebensereignisse

Niedtfeld, I. (Mannheim); Schmidt, A.F.; Scholz, O.B. (Bonn)

Originalarbeit - Kinder und Jugendliche

101 Effektivität kinderverhaltenstherapeutischer Maßnahmen in der Jugendhilfe: Das Training mit aggressiven Kindern

Petermann, U.; Petermann, F. (Bremen); Büttner, P.; Krause-Leipoldt, C. (Schlüchtern); Nitkowski, D. (Bremen)

\section{Übersichtsarbeit}

109 Stabilisierung vor Konfrontation in der Traumatherapie - Grundregel oder Mythos? Neuner, F. (Konstanz)

Interview

120 Helen Mayberg: «Therapieresistente Depression kann durch Tiefenhirnstimulation beeinflusst werden» Nater, U. (Zürich)
Vol. 18, Issue 2, June 2008

Obituary

72 Steven Karger (1959-2008)

Editorial

73 What's the Relevance of Alternative Treatment Approaches Other than Cognitive Behaviour Therapy? Ehlert, U. (Zürich)

\section{Original Articles}

75 Does a Manual-Based, Disorder-Focused Psychotherapy Reduce Comorbid Symptoms in Bulimia Nervosa?

Tuschen-Caffier, B. (Freiburg i.Br.); Pook, M. (München); Frank, M. (Marburg)

83 Beat-to-Beat Blood Pressure Biofeedback as a Supplementary Treatment for Arterial Hypertension Piesbergen, C. (München); Kircher, K. (Mühldorf/Inn); Weber, R. (Bernried)

91 How Does Expressive Writing Take Effect? Differential Mechanisms of Writing about Stressful Life Events

Niedtfeld, I. (Mannheim); Schmidt, A.F.; Scholz, O.B. (Bonn)

Original Article - Children and Adolescents

101 Effectiveness of Cognitive Behavioral Interventions for Children in Youth Welfare Services: Training with Aggressive Children

Petermann, U.; Petermann, F. (Bremen); Büttner, P.; Krause-Leipoldt, C. (Schlüchtern); Nitkowski, D. (Bremen)

Review Article

109 Stabilization before Confrontation in Trauma Treatment - Elementary Rule or Myth? Neuner, F. (Konstanz)

Interview

120 Helen Meyberg: 'Therapy-Resistant Depression Can Be Modulated Using Deep Brain Stimulation' Nater, U. (Zürich)

\begin{tabular}{ll}
\hline KARGER & $\oplus$ 2008 S. Karger GmbH, Freiburg \\
Fax +49 761 4520714 & Artikel (Volltext) und Inhaltsverzeichnisse \\
$\begin{array}{l}\text { E-mail Information@Karger.de } \\
\text { www.karger.com }\end{array}$ & $\begin{array}{l}\text { sowie das vorläufige Inhaltsverzeichnis des nächsten Heftes: } \\
\text { www.karger.com/ver_bk.htm }\end{array}$
\end{tabular}




\section{Verhaltenstherapie}

\section{Band 18, Heft 2, Juni 2008}

125 Buchbesprechungen

129 Fort- und Weiterbildung

133 Mitteilungen der Verbände

137 Tagungen und Kongresse

139 Hinweise für Autoren

74 Impressum

U2 Erläuterungen zum Titelbild (2. Umschlagseite) Leibl, C.; Austermann, T.; Naab, S.; Gautier, L. (Prien am Chiemsee)

Einen Ausblick auf den Inhalt der kommenden Hefte finden Sie auf Seite 140.

\section{Vol. 18, Issue 2, June 2008}

125 Books Reviews

129 Education

133 Information by Behavior Therapy Associations

137 Meetings and Conferences

139 Guidelines for Authors

74 Imprint

C2 Explanations on the Title (Inside front cover) Leibl, C.; Austermann, T.; Naab, S.; Gautier, L. (Prien am Chiemsee)

Forthcoming papers are listed on page 140. 